\title{
Universality and Shannon entropy of codon usage
}

\author{
L. Frappat, ${ }^{1, *}$ C. Minichini, ${ }^{2, \dagger}$ A. Sciarrino, ${ }^{2,3, \ddagger}$ and P. Sorba ${ }^{4, \S}$ \\ ${ }^{1}$ Laboratoire d'Annecy-le-Vieux de Physique Théorique LAPTH, CNRS, UMR 5108 associée à l'Université de Savoie, Boîte Postale 110, \\ F-74941 Annecy-le-Vieux Cedex, France \\ ${ }^{2}$ Dipartimento di Scienze Fisiche, Università di Napoli "Federico II," Naples, Italy \\ ${ }^{3}$ INFN, Sezione di Napoli, Complesso Universitario di Monte S. Angelo, Via Cintia, I-80126 Naples, Italy \\ ${ }^{4}$ CERN, Theory Division, CH-1211 Geneva 23, Switzerland
}

(Received 28 April 2003; published 24 December 2003)

\begin{abstract}
The distribution functions of codon usage probabilities, computed over all the available GenBank data for 40 eukaryotic biological species and five chloroplasts, are best fitted by the sum of a constant, an exponential, and a linear function in the rank of usage. For mitochondria the analysis is not conclusive. These functions are characterized by parameters that strongly depend on the total guanine and cytosine $(G C)$ content of the coding regions of biological species. It is predicted that the codon usage is the same in all exonic genes with the same $G C$ content. The Shannon entropy for codons, also strongly dependent on the exonic $G C$ content, is computed.
\end{abstract}

DOI: 10.1103/PhysRevE.68.061910

PACS number(s): 87.10.+e, 02.10.-v

\section{INTRODUCTION}

In the recent past, some interest has been shown in applying methods of statistical linguistics and information theory for the analysis of DNA sequences, ${ }^{1}$ in particular, in investigating whether the frequency distribution of nucleotides or sequences of nucleotides follows Zipf's law [1], and using the Shannon entropy to identify the redundancy or the bias of a nucleotide sequence. Let us recall that, at the end of the 1940s, Zipf remarked that, in natural languages and in many other domains, the distribution function follows an inverse power law, which can be described, denoting by rank $n=1$ the most used word, by $n=2$ the next one, and so on, and with $a>0$, by

$$
f_{n}=\frac{f_{1}}{n^{\alpha}}
$$

In $[2,3]$, it was claimed that noncoding sequences of DNA are more similar to natural languages than coding ones, and the Shannon entropy has been used to quantify the redundancy of words. This work raised a debate in the literature (see [4]). In particular, in [5] it was shown that the oligonucleotide frequencies in DNA, in both coding and noncoding sequences, follow a Yule and not a Zipf distribution. Let us recall that the Yule distribution with parameters $a, b, c$ $>0$ is given by [6]

\footnotetext{
*Email address: frappat@lapp.in2p3.fr

†Email address: minichini@na.infn.it

*Email address: sciarrino@na.infn.it

${ }^{\S}$ On leave of absence from LAPTH, Annecy-le-Vieux Cedex,

France. Email address: sorba@lapp.in2p3.fr; sorba@cern.ch

${ }^{1}$ DNA is constituted of four bases, adenine $(A)$, cytosine $(C)$, guanine $(G)$, and thymine $(T)$, this last one being replaced by uracile $(U)$ in messenger RNA. A codon is defined as an ordered sequence of three bases. Coding sequences in DNA are characterized by their constituent codons.
}

$$
f_{n}=c n^{-a} b^{n}
$$

Note that Zipf's law is observed from $n$ ranked random samples of $\chi^{2}$ distributed variables, as shown in [7]. In a recent work [8] it was argued that Zipf's law is well adapted to represent the abundance of expressed genes, with an exponent $a \approx 1$. However, in [9] the analyzed distributions of gene expressions are well fitted by a family of Pareto distributions.

Indeed, in the literature many have claimed that Zipf's laws are not really power laws. As the main point of our paper is not the analysis of the validity of this law, we will no longer pursue the debate, and we refer the interested reader to the web site on Zipf's law (http://linkage.rockefeller.edu/ wli/zipf/), where a large literature (updated to 2001) on the applications of this law in different domains can be found.

Recently, an analysis of the rank distribution for codons, performed in many genes for several biological species, led the authors of [10] to fit experimental data with an exponential function. In particular, by considering separately different coding DNA sequences, they studied the relation between the parameter in the exponential, the frequency of rank 1 , and the length of the sequence for different genes. From this very short overview, it follows that the determination of the kind of law followed by the codon rank distribution is extremely interesting in investigations of the nature of the evolutionary process, which has acted upon the codon distribution, i.e., the eventual presence of a bias.

In the last few years, the number of available data for coding sequences has considerably increased, but apparently no analysis using the whole set of data has been performed. Here we present the results of such a study. The main aim of this paper is to show the existence of a universal, i.e., biological species independent, distribution law for codons for the eukaryotic code. As a result of our investigation, we point out that the rank of codon usage probabilities follows a universal law, the frequency function of the rank- $n$ codon showing up as a sum of an exponential part and a linear part. Such a universal behavior suggests the presence of general biases, one of which is identified with the total exonic $G C$ 
TABLE I. Values of the best-fit parameters, Eq. (4), for the sample of biological species. Types: vrt vertebrates (6), inv $=$ invertebrates (3), pln $=$ plants (4), fng =fungi (2), bct=bacteria (25).

\begin{tabular}{|c|c|c|c|c|c|c|}
\hline Type & Species & $G C$ content $(\%)$ & $\alpha$ & $\eta$ & $10^{4} \beta$ & $\chi^{2}$ \\
\hline vrt & Homo sapiens & 52.58 & 0.0214 & 0.073 & 1.65 & 0.0126 \\
\hline pln & Arabidopsis thaliana & 44.55 & 0.0185 & 0.056 & 1.68 & 0.0051 \\
\hline inv & Drosophila melanogaster & 54.03 & 0.0247 & 0.081 & 1.67 & 0.0089 \\
\hline inv & Caenorhabditis elegans & 42.79 & 0.0216 & 0.064 & 1.79 & 0.0063 \\
\hline vrt & Mus musculus & 52.38 & 0.0208 & 0.071 & 1.57 & 0.0112 \\
\hline fng & Saccharomyces cervisiae & 39.69 & 0.0246 & 0.069 & 1.91 & 0.0127 \\
\hline bct & Escherichia coli & 50.52 & 0.0233 & 0.065 & 1.91 & 0.0112 \\
\hline vrt & Rattus norvegicus & 52.87 & 0.0222 & 0.073 & 1.63 & 0.0083 \\
\hline pln & Oryza sativa japonica & 55.84 & 0.0179 & 0.073 & 1.63 & 0.0211 \\
\hline fng & Schizosaccharomyces pombe & 39.80 & 0.0255 & 0.068 & 1.98 & 0.0036 \\
\hline bct & Bacillus subtilis & 44.32 & 0.0259 & 0.084 & 1.71 & 0.0241 \\
\hline bct & Pseudomonas aeruginosa & 65.70 & 0.0538 & 0.107 & 2.76 & 0.0191 \\
\hline bct & Mesorhizobium loti & 63.05 & 0.0416 & 0.093 & 2.44 & 0.0093 \\
\hline bct & Streptomyces coelicolor A3 & 72.41 & 0.0567 & 0.098 & 3.14 & 0.0456 \\
\hline bct & Sinorhizobium meliloti & 62.71 & 0.0359 & 0.076 & 2.54 & 0.0067 \\
\hline bct & Nostoc sp. PCC7120 & 42.36 & 0.0288 & 0.098 & 1.63 & 0.0140 \\
\hline pln & Oryza sativa & 54.63 & 0.0173 & 0.062 & 1.59 & 0.0135 \\
\hline bct & Agrobacterium tumefaciens str. C58 & 59.74 & 0.0308 & 0.067 & 2.43 & 0.0100 \\
\hline bet & Ralstonia solanacearum & 67.57 & 0.0543 & 0.105 & 2.87 & 0.0149 \\
\hline bet & Yersinia pestis & 48.97 & 0.0179 & 0.040 & 2.17 & 0.0066 \\
\hline bct & Methanosarcina acetivorans str. C24 & 45.17 & 0.0228 & 0.068 & 1.81 & 0.0214 \\
\hline bct & Vibrio cholerae & 47.35 & 0.0203 & 0.052 & 2.02 & 0.0100 \\
\hline bct & Escherichia coli $\mathrm{K} 12$ & 51.83 & 0.0250 & 0.065 & 2.05 & 0.0117 \\
\hline bct & Mycobacterium tuberculosis CDC1551 & 65.77 & 0.0401 & 0.094 & 2.35 & 0.0105 \\
\hline bct & Mycobacterium tuberculosis H87Rv & 65.90 & 0.0414 & 0.097 & 2.29 & 0.0109 \\
\hline bct & Bacillus halodurans & 44.32 & 0.0263 & 0.100 & 1.27 & 0.0233 \\
\hline bct & Clostridium acetobutylicum & 31.59 & 0.0434 & 0.087 & 2.76 & \\
\hline bct & Caulobacter crescentus CB15 & 67.68 & 0.0570 & 0.113 & 2.86 & 0.0087 \\
\hline vrt & Gallus gallus & 52.11 & 0.0239 & 0.095 & 1.17 & 0.0129 \\
\hline bct & Synechocystis sp. PCC6803 & 48.56 & 0.0260 & 0.083 & 1.49 & 0.0140 \\
\hline bct & Sulfolobulus solfataricus & 36.47 & 0.0290 & 0.066 & 2.26 & 0.0099 \\
\hline bct & Mycobacterium leprae & 59.90 & 0.0252 & 0.071 & 1.80 & 0.0065 \\
\hline bct & Brucella melitensis & 58.25 & 0.0294 & 0.067 & 2.25 & 0.0121 \\
\hline bct & Deinococcus radiodurans & 67.24 & 0.0481 & 0.098 & 2.76 & 0.0113 \\
\hline vrt & Xenopus laevis & 47.33 & 0.0193 & 0.084 & 0.92 & 0.0268 \\
\hline bct & Listeria monocytogenens & 38.39 & 0.0437 & 0.136 & 1.64 & 0.0267 \\
\hline pln & Neurospora crassa & 56.17 & 0.0241 & 0.086 & 1.31 & 0.0166 \\
\hline bct & Clostridium perfrigens & 29.47 & 0.0510 & 0.092 & 3.11 & \\
\hline inv & Leishmania major & 63.36 & 0.0294 & 0.069 & 2.21 & 0.0050 \\
\hline vrt & Bos taurus & 53.05 & 0.0240 & 0.089 & 1.27 & 0.0126 \\
\hline
\end{tabular}

content. Indeed, the values of the parameters appearing in the fitting expression are plotted versus the total percentage of exonic $G C$ content of the biological species and are reasonably well fitted by a parabola. Finally, from the expression obtained, we derive the theoretical prediction that the usage probability for rank-ordered codons is the same in any gene region having the same exonic $G C$ content for any biological species.

We compute the Shannon entropy [11] for amino acids and find that its behavior as a function of the exonic $G C$ content is also a parabola, whose apex is around the value 0.50 of the $G C$ content.

\section{CODON USAGE PROBABILITY DISTRIBUTION}

Let us define the usage probability for the codon $X Z N$ $(X, Z, N \in\{A, C, G, U\})$ as

$$
P(X Z N)=\lim _{n_{\mathrm{tot}} \rightarrow \infty} \frac{n_{X Z N}}{N_{\text {tot }}}
$$

where $n_{X Z N}$ is the number of times the codon $X Z N$ has been used in the analyzed biosynthesis process for a given biological species, and $N_{\text {tot }}$ is the total number of codons used in all processes considered. It follows that our analysis and predic- 


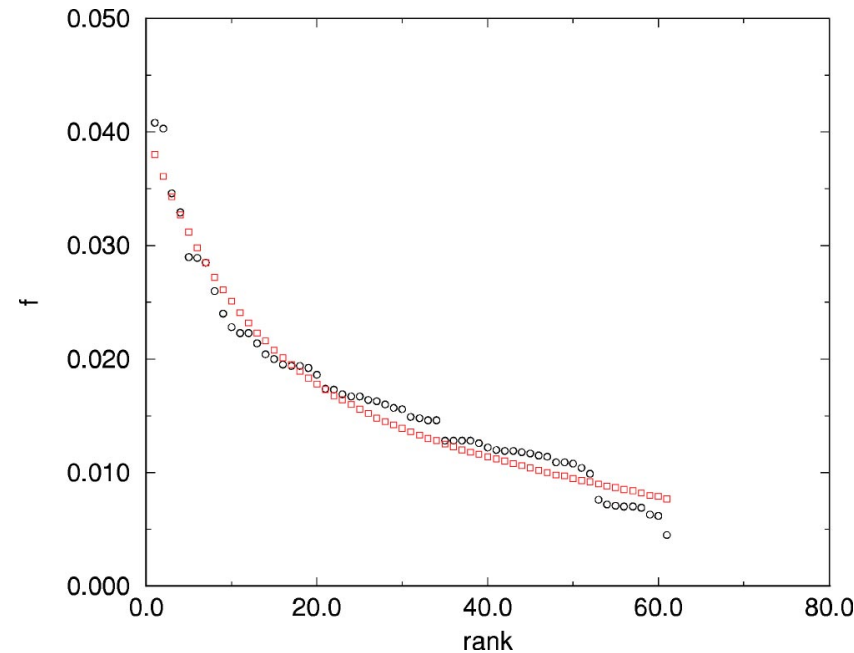

FIG. 1. Rank distribution of the codon usage probabilities for Homo sapiens. Circles are experimental values, squares are fitted values.

tions hold for biological species with sufficiently large statistics of codons. For each biological species, codons are ordered following decreasing order of the values of their usage probabilities, i.e., codon number 1 corresponds to the highest value, codon number 2 is the next highest, and so on. We denote by $f(n)$ the probability $P(X Z N)$ of finding that $X Z N$ is in the $n$th position. Of course the same codon occupies in general two different positions in the rank distribution function for two different species. We plot $f(n)$ versus the rank and we determine that the data are well fitted by the sum of an exponential function, a linear function in the rank, and a constant, i.e.,

$$
f(n)=\alpha e^{-\eta n}-\beta n+\gamma,
$$

where $0.0187 \leqslant \alpha \leqslant 0.0570, \quad 0.050 \leqslant \eta \leqslant 0.136, \quad 0.82 \times 10^{-4}$ $\leqslant \beta \leqslant 3.63 \times 10^{-4}$, and $\gamma=0.016$ are constant depending on the biological species. These four constants have to satisfy the normalization condition

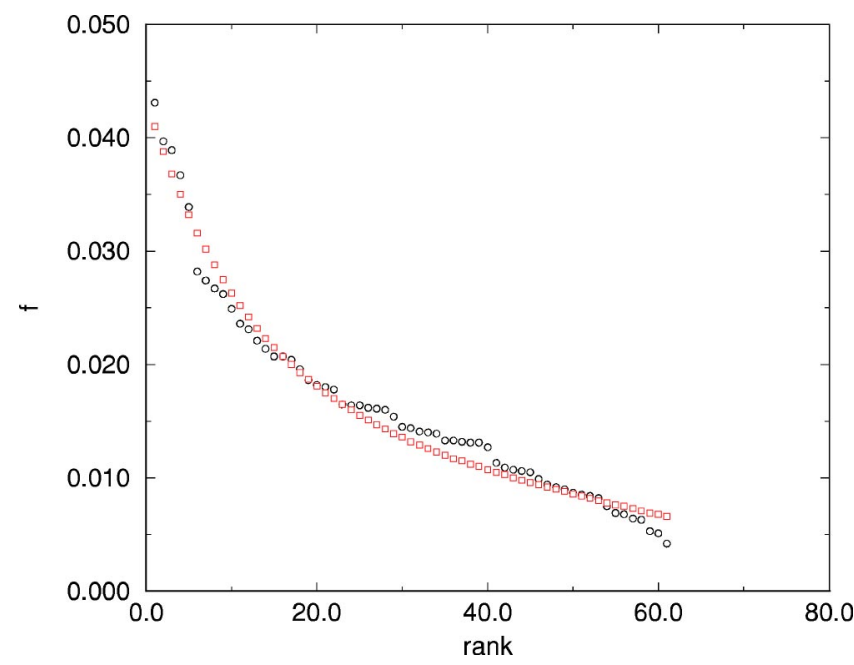

FIG. 2. Rank distribution of the codon usage probabilities for Drosophila melanogaster. Circles are experimental values, squares are fitted values.

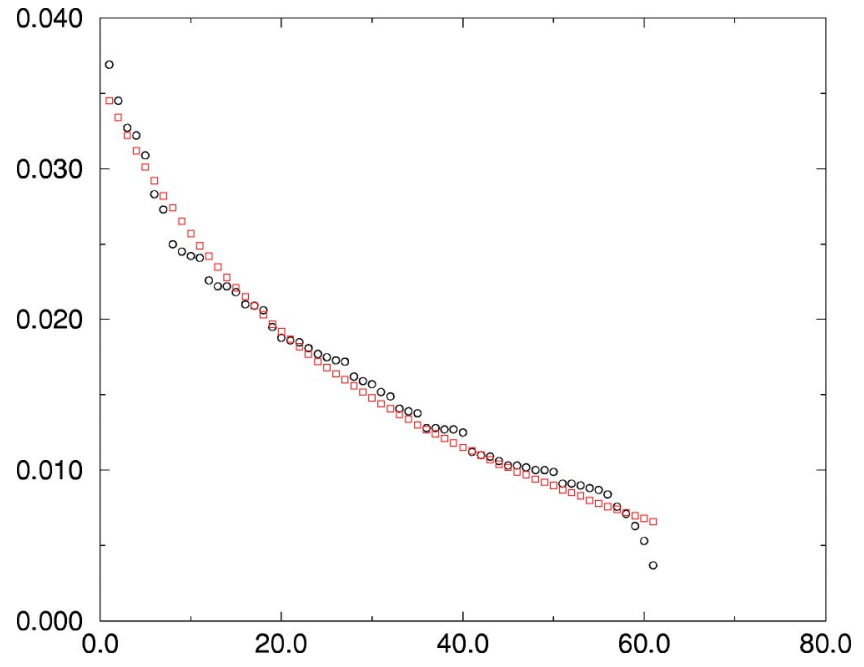

FIG. 3. Rank distribution of the codon usage probabilities for Arabidopsis thaliana. Circles are experimental values, squares are fitted values.

$$
\sum_{n} f(n)=1
$$

In Table I we list the 40 biological species (six vertebrates, four plants, three invertebrates, two fungi and 25 bacteria) with a sample of codons of sizes between 800000 and 20000000 in decreasing order (data from GenBank release 129.0 [12]) whose codon usage has been fitted, specifying for each biological species the value of the parameters computed by a best-fit procedure and the corresponding $\chi^{2}$. Here and in the following, the $\chi^{2}$ coefficient is defined by

$$
\chi^{2}=\sum_{i} \frac{\left[y_{i}-y\left(x_{i}\right)\right]^{2}}{y\left(x_{i}\right)}
$$

where $x_{i}$ are the experimental abscissae, $y_{i}$ the experimental values, and $y\left(x_{i}\right)$ the fitted ones. In some cases, $y\left(x_{i}\right)$ takes

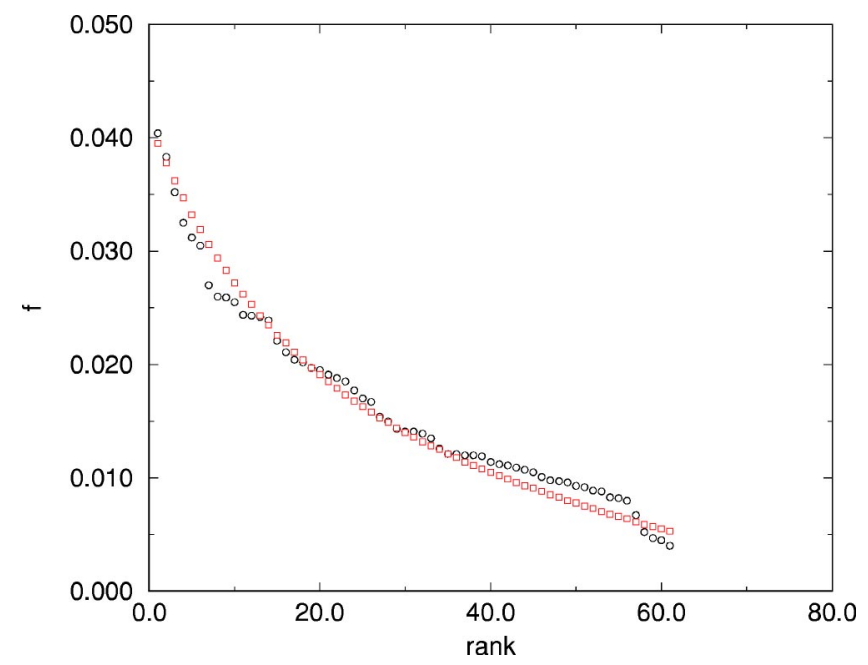

FIG. 4. Rank distribution of the codon usage probabilities for Escherichia coli. Circles are experimental values, squares are fitted values. 
TABLE II. Type of codons used for the observed rank distribution $f(n)$.

\begin{tabular}{|c|c|c|c|c|c|c|c|c|c|c|c|c|c|c|c|c|c|c|c|c|}
\hline \multirow[b]{2}{*}{ Species } & \multicolumn{20}{|c|}{ Rank } \\
\hline & 1 & 2 & 3 & 4 & 5 & 6 & 7 & 8 & 9 & 10 & 11 & 12 & 13 & 14 & 15 & 16 & 17 & 18 & 19 & 20 \\
\hline Homo sapiens & $G A G$ & $C U G$ & $C A G$ & $A A G$ & $G A A$ & $G U G$ & $G C C$ & $G A C$ & $A A A$ & $G G C$ & $A U G$ & $G A U$ & $A U C$ & $U U C$ & $C C C$ & $A A C$ & $C U C$ & $A G C$ & $A C C$ & $G C U$ \\
\hline Mus musculus & $C U G$ & $G A G$ & $A A G$ & $C A G$ & $G U G$ & $G A C$ & $G A A$ & $G C C$ & $A U C$ & $A U G$ & $G G C$ & $U U C$ & $G A U$ & $A A A$ & $A A C$ & $C U C$ & $G C U$ & $A G C$ & $A C C$ & $C C C$ \\
\hline Rattus norvegicus & $C U G$ & $G A G$ & $A A G$ & $C A G$ & $G U G$ & $G A C$ & $G C C$ & $G A A$ & $A U C$ & $U U C$ & $A U G$ & $A A C$ & $G G C$ & $C U C$ & $G A U$ & $A A A$ & $A C C$ & $A G C$ & $G C U$ & $C C C$ \\
\hline Gallus gallus & $G A G$ & $C U G$ & $A A G$ & $C A G$ & $G A A$ & $G U G$ & $A A A$ & $G A C$ & $G C C$ & $G A U$ & $A A C$ & $A U C$ & $A U G$ & $G G C$ & $A G C$ & $U U C$ & $G C U$ & $C C C$ & $U A C$ & $A C C$ \\
\hline Xenopus laevis & GAA & $G A G$ & $A A A$ & $A A G$ & $C A G$ & $G A U$ & $C U G$ & $A U G$ & $G A C$ & $A A U$ & $A A C$ & $G G A$ & $G U G$ & $G C U$ & $C C A$ & $A U U$ & $G C A$ & $U U U$ & $U G U$ & $A G A$ \\
\hline Bos taurus & $C U G$ & $G A G$ & $A A G$ & $C A G$ & $G U G$ & $G C C$ & $G A C$ & $G A A$ & $A U C$ & $G G C$ & $U U C$ & $A A C$ & $A U G$ & $A A A$ & $A C C$ & $G A U$ & $C U C$ & $C C C$ & $U A C$ & $A G C$ \\
\hline Arabidopsis thaliana & $G A U$ & $G A A$ & $A A G$ & $G A G$ & $A A A$ & $G C U$ & $G U U$ & $U C U$ & $A U G$ & $C U U$ & $G G A$ & $A A U$ & $G G U$ & $U U U$ & $A U U$ & $U U G$ & $A A C$ & $U U C$ & $C A A$ & $A G A$ \\
\hline Oryza sativa japonica & $G A G$ & $G C C$ & $G G C$ & $A A G$ & $G A C$ & $G C G$ & $C U C$ & $G U G$ & $G A U$ & $A U G$ & $U U C$ & $C U G$ & $G A A$ & $C A G$ & $G U C$ & $A U C$ & $C C G$ & $G C U$ & $A A C$ & $C G C$ \\
\hline Oryza sativa & $G A G$ & $A A G$ & $G C C$ & $G G C$ & $G A C$ & $G A U$ & $C U C$ & $A U G$ & $G U G$ & $G C G$ & $U U C$ & $C A G$ & $G A A$ & $A U C$ & $G C U$ & $A A C$ & $G U C$ & $C U G$ & $G C A$ & $G G G$ \\
\hline Neurospora crassa & $G A G$ & $A A G$ & $G C C$ & $G A C$ & $G G C$ & $A A C$ & $C U C$ & $A U C$ & $C A G$ & $G U C$ & $A C C$ & $G A U$ & $C C C$ & $U U C$ & $A U G$ & $G A A$ & $G C U$ & $U C C$ & $G G U$ & $U A C$ \\
\hline Drosophila melanogaster & $G A G$ & $A A G$ & $C U G$ & $C A G$ & $G C C$ & $G U G$ & $G A U$ & $G G C$ & $A A C$ & $G A C$ & $A U G$ & $A U C$ & $U U C$ & $A C C$ & $G A A$ & $A A U$ & $A G C$ & $U C C$ & $U A C$ & $C G C$ \\
\hline Caenorhabditis elegans & GAA & $A A A$ & $G A U$ & $A U U$ & $G G A$ & $A A U$ & $C A A$ & $A U G$ & $A A G$ & $C C A$ & $U U U$ & $U U C$ & $G A G$ & $G U U$ & $G C U$ & $C U U$ & $U C A$ & $U U G$ & $A C A$ & $G C A$ \\
\hline Leishmania major & $G C G$ & $G A G$ & $G C C$ & $C U G$ & $G U G$ & $G G C$ & $G A C$ & $C A G$ & $C G C$ & $A A G$ & $C C G$ & $A G C$ & $C U C$ & $A C G$ & $A U G$ & $A A C$ & $U C G$ & $C A C$ & $G C A$ & $U A C$ \\
\hline Sacch. cerevisiae & $G A A$ & $A A A$ & $G A U$ & $A A U$ & $A A G$ & $A U U$ & $C A A$ & $U U G$ & $U U A$ & $U U U$ & $A A C$ & $G G U$ & $U C U$ & $G U U$ & $A G A$ & $G C U$ & $A U G$ & $G A C$ & $A C U$ & $G A G$ \\
\hline Schizosacch. pombe & GAA & $A A A$ & $G A U$ & $A U U$ & $A A U$ & $U U U$ & $U C U$ & $G C U$ & $G U U$ & $C A A$ & $U U A$ & $C U U$ & $A A G$ & $U U G$ & $A C U$ & $U A U$ & $C C U$ & $G G U$ & $G A G$ & $A U G$ \\
\hline Escherichia coli & $C U G$ & $G A A$ & $A A A$ & $G A U$ & $G C G$ & $A U U$ & $C A G$ & $G G C$ & $A U G$ & $G G U$ & $G U G$ & $G C C$ & $A U C$ & $U U U$ & $A C C$ & $A A C$ & $G C A$ & $C C G$ & $A A U$ & $C G U$ \\
\hline Bacillus subtilis & $A A A$ & $G A A$ & $A U U$ & $G A U$ & $U U U$ & $A U C$ & $A U G$ & $G G C$ & $G A G$ & $C U G$ & $C U U$ & $U A U$ & $A A U$ & $A C A$ & $G G A$ & $G C A$ & $A A G$ & $G C G$ & $C A A$ & $U U A$ \\
\hline Pseudom. aeruginosa & $C U G$ & $G C C$ & $G G C$ & $C G C$ & $G A C$ & $G C G$ & $A U C$ & $G A G$ & $C A G$ & $G U G$ & $U U C$ & $A C C$ & $C C G$ & $G U C$ & $C U C$ & $A A G$ & $A G C$ & $G A A$ & $A A C$ & $A U G$ \\
\hline Mesorhizobium loti & $G G C$ & $G C C$ & $C U G$ & $A U C$ & $G C G$ & $G U C$ & $G A C$ & $C G C$ & $U U C$ & $G A G$ & $C C G$ & $A A G$ & $C U C$ & $G U G$ & $A C C$ & $C A G$ & $A U G$ & $G A A$ & $U C G$ & $G A U$ \\
\hline Streptom. coelicolon A3 & $G C C$ & $G G C$ & $C U G$ & $G A C$ & $G C G$ & $G A G$ & $G U C$ & $A C C$ & $C G C$ & $C U C$ & $G U G$ & $C C G$ & $C G G$ & $A U C$ & $U U C$ & $C C C$ & $C A G$ & $C A C$ & $U C C$ & $A A G$ \\
\hline Sinorhizobium meliloti & $G G C$ & $G C C$ & $G C G$ & $A U C$ & $G U C$ & $C U C$ & $C U G$ & $G A C$ & $C G C$ & $G A G$ & $U U C$ & $C C G$ & $A A G$ & $G A A$ & $A U G$ & $C A G$ & $G U G$ & $A C C$ & $A C G$ & $U C G$ \\
\hline Nostoc, sp. PCC7120 & GAA & $A U U$ & $C A A$ & $U U A$ & $A A A$ & $G A U$ & $A A U$ & $U U U$ & $G C U$ & $G G U$ & $G C A$ & $U U G$ & $G U U$ & $A C U$ & $U A U$ & $G U A$ & $A C A$ & $A U C$ & $G C C$ & $A U G$ \\
\hline Agrobact. tumefaciens & $G C C$ & $G G C$ & $C U G$ & $A U C$ & $G C G$ & $G A A$ & $C G C$ & $G U C$ & $U U C$ & $G A U$ & $G A C$ & $A A G$ & $C U C$ & $C C G$ & $A U G$ & $G U G$ & $C A G$ & $G A G$ & $A C C$ & $A C G$ \\
\hline Ralstonia solanacearum & $C U G$ & $G C C$ & $G G C$ & $G C G$ & $C G C$ & $G U G$ & $A U C$ & $G A C$ & $C C G$ & $C A G$ & $G A G$ & $U U C$ & $A C C$ & $G U C$ & $A A G$ & $A C G$ & $A U G$ & $A A C$ & $U C G$ & $C U C$ \\
\hline Yersinia pestis & $C U G$ & $G A U$ & $G A A$ & $A A A$ & $A U U$ & $G C C$ & $A U G$ & $G G U$ & $C A G$ & $A A U$ & $G C G$ & $G G C$ & $C A A$ & $A U C$ & $U U G$ & $G U G$ & $U U U$ & $A C C$ & $U U A$ & $G A G$ \\
\hline Methanosarc. acetivorans & $G A A$ & $A A A$ & $C U U$ & $G A U$ & $G G A$ & $A U U$ & $G C A$ & $A U C$ & $G A G$ & $U U U$ & $C U G$ & $G A C$ & $A U G$ & $A A U$ & $A A G$ & $A A C$ & $A U A$ & $G U U$ & $U A U$ & $U U C$ \\
\hline Vibrio cholerae & $G A A$ & $G A U$ & $A A A$ & $C A A$ & $A U U$ & $G C G$ & $G U G$ & $U U U$ & $C U G$ & $G G U$ & $A U G$ & $A U C$ & $G A G$ & $G G C$ & $U U G$ & $A A U$ & $G C C$ & $U U A$ & $G C U$ & $A C C$ \\
\hline Escherichia coli $\mathrm{K} 12$ & $C U G$ & $G A A$ & $G C G$ & $A A A$ & $G A U$ & $A U U$ & $G G C$ & $C A G$ & $A U G$ & $G U G$ & $G C C$ & $A U C$ & $G G U$ & $A C C$ & $C C G$ & $U U U$ & $C G C$ & $A A C$ & $C G U$ & $G C A$ \\
\hline Mycobact. tuber. CDC1551 & $G C C$ & $C U G$ & $G G C$ & $G C G$ & $G A C$ & $G U G$ & $A C C$ & $A U C$ & $G U C$ & $C C G$ & $G A G$ & $C G C$ & $C G G$ & $C A G$ & $U U C$ & $U C G$ & $A A C$ & $G G G$ & $G G U$ & $A U G$ \\
\hline Mycobact. tuber. H37Rv & $G C C$ & $G G C$ & $C U G$ & $G C G$ & $G A C$ & $G U G$ & $A C C$ & $A U C$ & $G U C$ & $C C G$ & $G A G$ & $C G C$ & $C G G$ & $U U C$ & $C A G$ & $A A C$ & $U C G$ & $G G G$ & $G G U$ & $A U G$ \\
\hline Bacillus. halodurans & $G A A$ & $A U U$ & $A A A$ & $G A U$ & $U U U$ & $G A G$ & $C A A$ & $U U A$ & $A U G$ & $A U C$ & $U A U$ & $C U U$ & $G G A$ & $G U U$ & $A A G$ & $A C G$ & $A A U$ & $G C A$ & $G U G$ & $G C G$ \\
\hline Clostridium acetobutylicum & $A A A$ & $A U A$ & $A A U$ & $G A A$ & $G A U$ & $U U U$ & $U U A$ & $A U U$ & $U A U$ & $G G A$ & $A A G$ & $G U U$ & $G U A$ & $C U U$ & $G C A$ & $A U G$ & $A G A$ & $G C U$ & $A C A$ & $G G U$ \\
\hline Caulobacter crescentus CB15 & GCC & $C U G$ & $G G C$ & $G C G$ & $G A C$ & $C G C$ & $A U C$ & $G U C$ & $G A G$ & $A C C$ & $A A G$ & $U U C$ & $G U G$ & $C C G$ & $C A G$ & $A U G$ & $U C G$ & $A A C$ & $C C C$ & $C U C$ \\
\hline Synechocystis sp. PCC6803 & $G A A$ & $A U U$ & $G C C$ & $C A A$ & $G A U$ & $U U G$ & $A A A$ & $U U U$ & $G U G$ & $A C C$ & $U U A$ & $C C C$ & $A A U$ & $G G C$ & $C A G$ & $C U G$ & $G C U$ & $G G U$ & $A U G$ & $G A C$ \\
\hline Sulfolobus solfataricus & $A U A$ & $U U A$ & $A A A$ & $G A A$ & $A A G$ & $G A U$ & $A U U$ & $A A U$ & $U A U$ & $G A G$ & $G U A$ & $G U U$ & $U U U$ & $G G A$ & $A G A$ & $G C U$ & $G G U$ & $A U G$ & $A C U$ & $G C A$ \\
\hline Mycobacterium leprae & $G C C$ & $C U G$ & $G U G$ & GAC & $G C G$ & $G G C$ & $A U C$ & $G U C$ & $A C C$ & $G A G$ & $C C G$ & $U U G$ & $G G U$ & $C G C$ & $C A G$ & $G A U$ & $G A A$ & $G C U$ & $U U C$ & $C G G$ \\
\hline Brucella melitensis & $G G C$ & $G C C$ & $C U G$ & $G A A$ & $C G C$ & $G C G$ & $A U C$ & $G A U$ & $A A G$ & $G U G$ & $C C G$ & $U U C$ & $A U G$ & $C A G$ & $C U U$ & $G A C$ & $G U C$ & $A C C$ & $C U C$ & $G A G$ \\
\hline Deinococcus radiodurans & $C U G$ & $G C C$ & $G G C$ & $G U G$ & $G C G$ & $G A C$ & $C G C$ & $A C C$ & $C A G$ & $C U C$ & $G A G$ & $C C C$ & $G A A$ & $C C G$ & $A G C$ & $G U C$ & $A U C$ & $U U C$ & $G G G$ & $C G G$ \\
\hline Listeria monocytogenes & $A A A$ & $G A A$ & $A U U$ & $G A U$ & $U U A$ & $A A U$ & $U U U$ & $C A A$ & $G C A$ & $G U U$ & $A U G$ & $A C A$ & $G G U$ & $U A U$ & $G C U$ & $G U A$ & $C U U$ & $G G A$ & $A U C$ & $C C A$ \\
\hline Clostridium perfringens & $A A A$ & $G A A$ & $U U A$ & $A U A$ & $A A U$ & $G A U$ & $G G A$ & $U U U$ & $G U U$ & $U A U$ & $A U U$ & $G C U$ & $A G A$ & $A A G$ & $G U A$ & $A U G$ & $A C U$ & $U C A$ & $G C A$ & $A C A$ \\
\hline
\end{tabular}




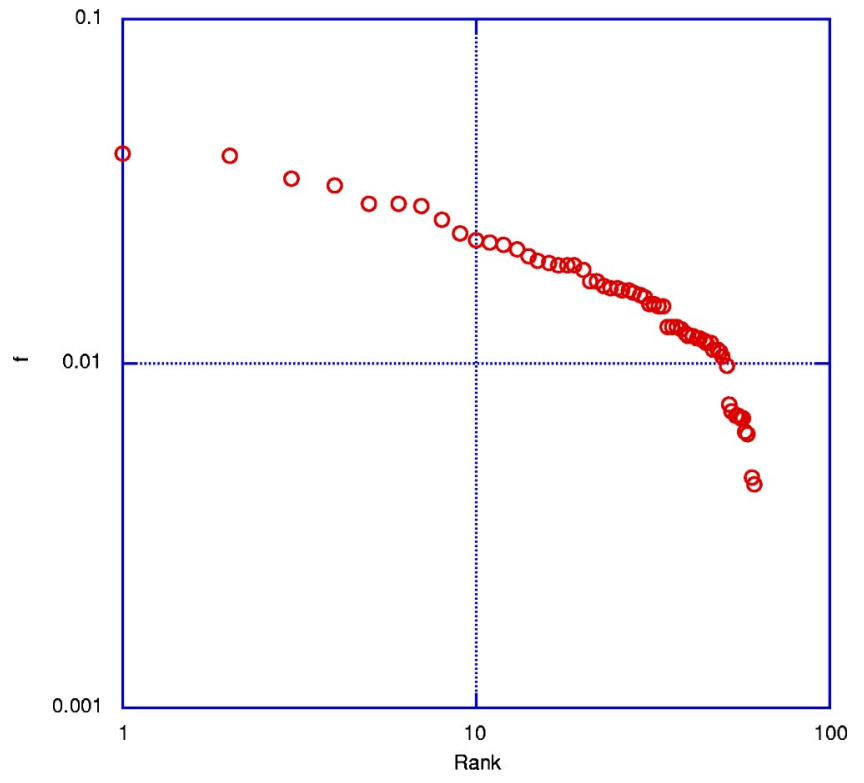

FIG. 5. Log-log ranked distribution of the codon usage probabilities for Homo sapiens.

vanishing or negative values for a few points and hence the $\chi^{2}$ is not reported. In Figs. $1-4$, we report the plots of $f(n)$ as a function of $n$ for a few biological species (Homo sapiens, Drosophila melanogaster, Arabidopsis thaliana, and Escherichia coli). The plot has been cut to $n=61$ to take into account the fact that in standard code there are three Stop codons (to end the biosynthesis process), whose function is very peculiar. For the same reason, the $\chi^{2}$ has been computed by taking into account the 61 coding codons only. In Table II, we report the type of the 20 most used codons of the observed rank distribution $f(n)$. The goodness of fit can be estimated by $P\left(n / 2, \chi^{2} / 2\right)$, where $P(a, x)$ is the incomplete Gamma function and $n$ is the number of degrees of freedom. $P\left(n / 2, \chi^{2} / 2\right)$ is the probability that the observed $\chi^{2}$ for a correct model should be less than the calculated $\chi^{2}$. In the present case, $P\left(n / 2, \chi^{2} / 2\right)$ is less than $10^{-5}$ for each species. In Fig. 5, for Homo sapiens, we draw the log-log ranked plot, which obviously does not show a linear trend taking into account all the points, as would be the case for a Zipf's law behavior. Indeed, as emphasized in [5], when the majority of points reside in the tail of the distribution, it is necessary to fit the whole range of data.

A similar study, for a sample of 20 vertebrates with codon statistics larger than 100000 , reveals that, for almost all bio-

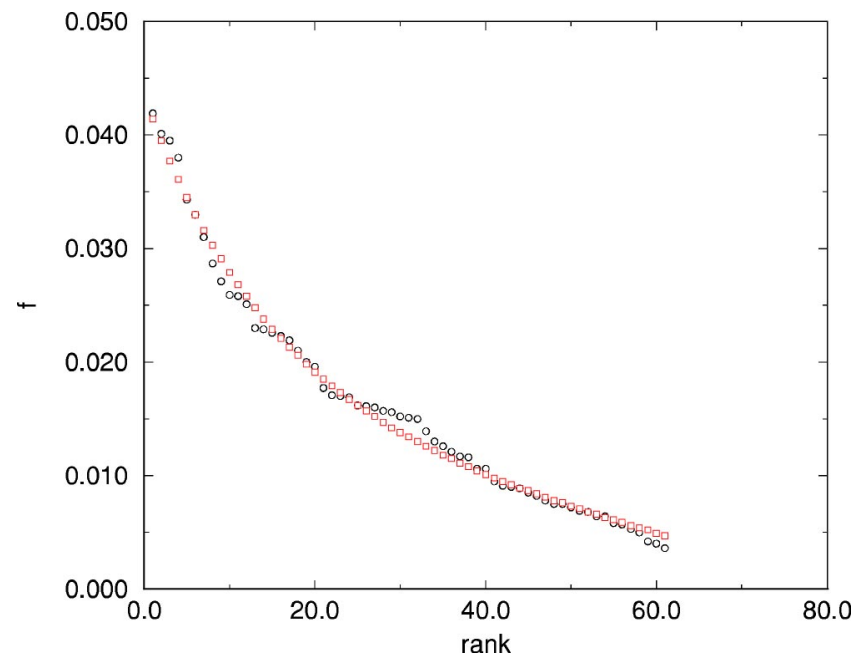

FIG. 6. Rank distribution of the codon usage probabilities for chloroplast Arabidopsis thaliana. Circles are experimental values, squares are fitted values.

logical species, the four most used codons are $G A G, C U G$, $A A G$, and $C A G$. All these codons have a $G$ nucleotide in the third position and three of them encode doublets. An analysis performed on the chloroplast codon usage for a sample of five plants gives the same result for the rank distribution $f(n)$; see Table III and Fig. 6 (Chloroplast Arabidopsis thaliana). We also report, in Table IV, the values of the parameters and the $\chi^{2}$ for a sample of nine mitochondria with codon statistics larger than 15000 . The fits for Homo sapiens and Arabidopsis thaliana are presented in Figs. 7 and 8. We point out, however, that for mitochondria the codon usage frequency distribution for several species (e.g., Arabidopsis thaliana or Drosophila melanogaster) is ill fitted by Eq. (4). This may be an indication that mitochondria do not follow the universal law (4). Note that the mitochondrial codes have a few differences from the eukaryotic code and vary slightly between species; see, e.g., [13]. In these cases, the $\chi^{2}$ has been computed over the corresponding coding codons. The value of the constant $\gamma$ is approximately equal to $1 / 61$ $=0.0164$ or $1 / 64=0.0156$, i.e., the value of the codon usage probability in the case of a uniform and unbiased codon distribution. Therefore the other two terms in Eq. (4) can be viewed as the effect of the bias mechanism. The appearance of the linear term is more intriguing. Let us remark that in [10], where an exponential function is used to fit the rank of usage in genes (not the rank of usage probability), the linear

TABLE III. Values of the best-fit parameters, Eq. (4), for the sample of chloroplasts.

\begin{tabular}{lccccc}
\hline \hline Species & GC content $(\%)$ & $\alpha$ & $\eta$ & $10^{4} \beta$ & $\chi^{2}$ \\
\hline Arabidopsis thaliana & 38.37 & 0.0254 & 0.067 & 1.95 & 0.0030 \\
Chaetosphaeridium globosum & 30.29 & 0.0515 & 0.110 & 2.59 & 0.0174 \\
Chlorella vulgaris & 34.63 & 0.0513 & 0.114 & 2.04 & 0.0093 \\
Cyanidium caldarium & 33.31 & 0.0379 & 0.092 & 2.24 & 0.0103 \\
Guillardia theta & 33.20 & 0.0452 & 0.103 & 2.20 & 0.0089 \\
\hline \hline
\end{tabular}


TABLE IV. Values of the best-fit parameters, Eq. (4), for the sample of mitochondria.

\begin{tabular}{ccccccc}
\hline \hline Type & Species & $G C$ content $(\%)$ & $\alpha$ & $\eta$ & $10^{4} \beta$ & $\chi^{2}$ \\
\hline vrt & Homo sapiens & 44.99 & 0.0414 & 0.099 & 2.31 & 0.0207 \\
pln & Arabidopsis thaliana & 44.18 & 0.0136 & 0.049 & 1.39 & 0.0589 \\
vrt & Mus musculus & 37.23 & 0.0455 & 0.104 & 2.44 & 0.0226 \\
fng & Saccharomyces cerevisiae & 24.17 & 0.0879 & 0.198 & 2.66 & 0.0611 \\
inv & Physarum polycephalum & 25.69 & 0.0624 & 0.128 & 2.70 & 0.0262 \\
pln & Pylaiella littoralis & 37.06 & 0.0336 & 0.108 & 1.72 & 0.0112 \\
pln & Neurospora crassa & 33.20 & 0.0388 & 0.101 & 2.14 & 0.0225 \\
vrt & Bos taurus & 39.73 & 0.0422 & 0.106 & 2.25 & 0.0430 \\
vrt & Sus scrofa & 40.52 & 0.0497 & 0.112 & 2.51 & 0.0372 \\
\hline \hline
\end{tabular}

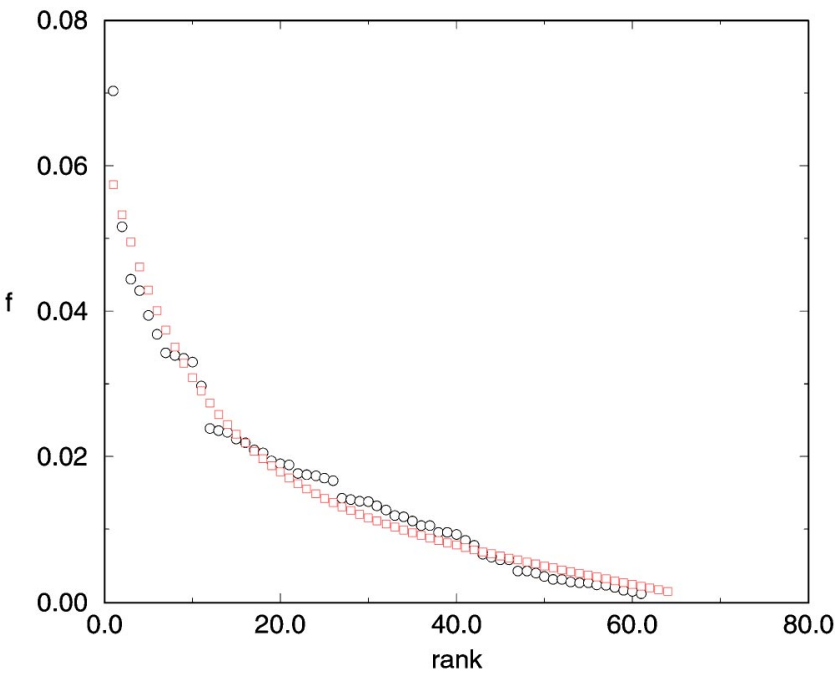

FIG. 7. Rank distribution of the codon usage probabilities for mitochondrial Homo sapiens. Circles are experimental values, squares are fitted values.

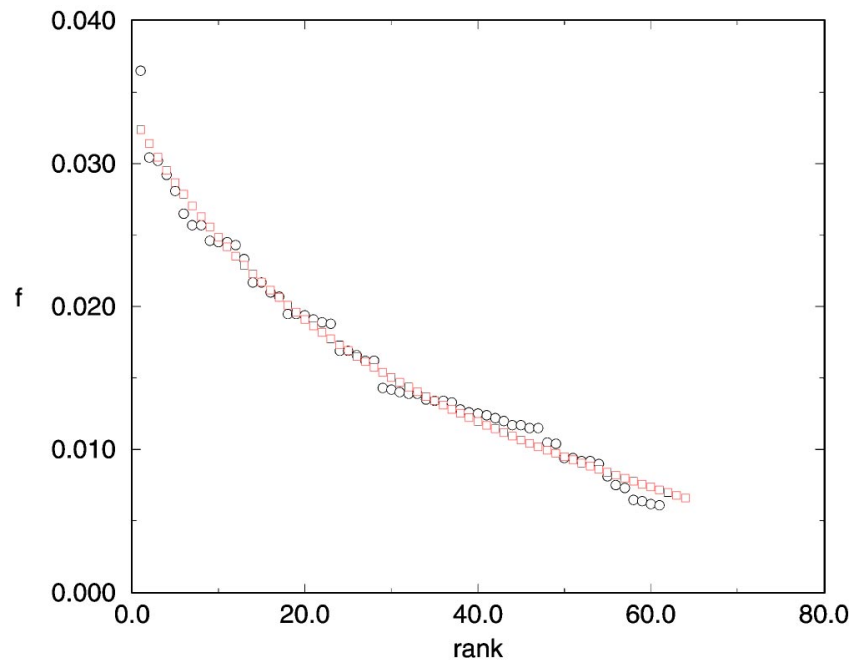

FIG. 8. Rank distribution of the codon usage probabilities for mitochondrial Arabidopsis thaliana. Circles are experimental values, squares are fitted values. term was observed, as its contribution becomes noticeable for approximately $n \geqslant 20$. Owing to the analysis of genes (with at most a few hundred codons), the fits in that paper end before this value of the rank. It is believed that the main causes of codon usage bias are translational efficiency, selection pressure, and spontaneous mutations. From the smallness of the parameter $\beta$ in Eq. (4), it is tempting to identify it as a consequence of the mutation effect and the first term in Eq. (4) as the effect of selection pressure, i.e., the interaction with the environment.

Since it is well known that the $G C$ content plays a strong role in the evolutionary process, we expect the parameters to depend on the total $G C$ content of the gene region (here the total exonic $G C$ content) that is indeed correlated with the evolution of the system (see [14] and references therein). We have investigated this dependence and report, in Fig. 9, the fits of $\alpha$ and $\beta$ to the total exonic $G C$ content $Y_{G C}$ of the biological species. One finds that the values of $\alpha$ and $\beta$ are well fitted by polynomial functions (with $0 \leqslant Y_{G C} \leqslant 100 \%$ ):

$$
\begin{aligned}
& \alpha=0.21145-0.00776 Y_{G C}+7.92 \times 10^{-5} Y_{G C}^{2}, \quad \chi^{2}=0.0262, \\
& \begin{aligned}
10^{2} \beta & =0.10096-0.00345 Y_{G C}+3.50 \times 10^{-5} Y_{G C}^{2}, \\
& =0.0170 .
\end{aligned}
\end{aligned}
$$

The two parameters $\alpha$ and $\beta$ appear to be correlated. Indeed the plot representing $\beta$ as a function of $\alpha$ is satisfactorily fitted by a regression line (see Fig. 10):

$$
10^{2} \beta=0.00851+0.375 \alpha, \quad \chi^{2}=0.0218 .
$$

The value of the $\eta$ parameter is largely uncorrelated with the total exonic $G C$ content. Let us recall, however, that $\eta$ is a function of $\alpha$ and $\beta$ due to the normalization condition of Eq. (5). Indeed we have ${ }^{2}$ (assuming $e^{-65 \eta} \approx 0$ )

$$
1=\frac{\alpha e^{-\eta}}{1-e^{-\eta}}+2080 \beta+64 \gamma
$$

\footnotetext{
${ }^{2}$ Note that the result is almost unchanged if the data are normalized on the 61 coding codons.
} 

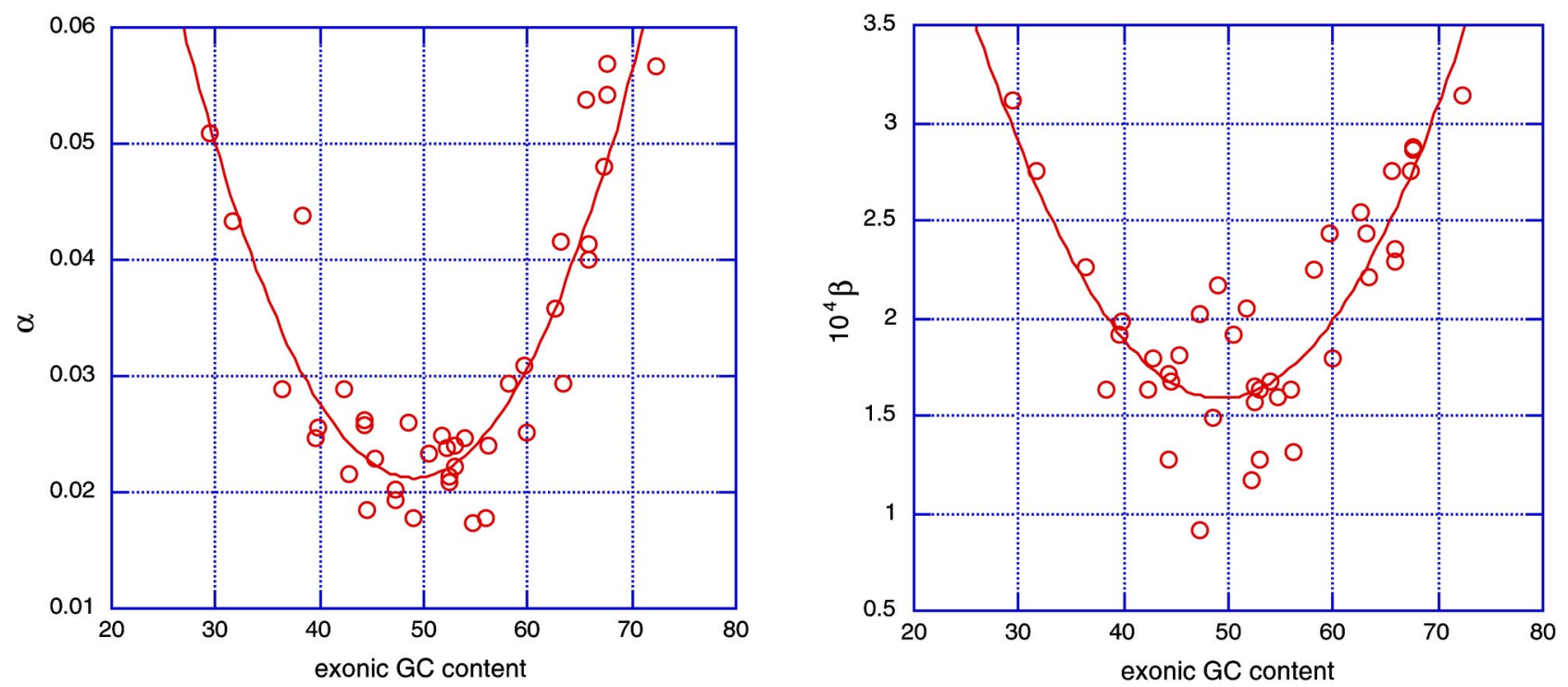

FIG. 9. Fits for the $\alpha$ and $\beta$ parameters.

Using the fits for $\alpha$ and $\beta$, we can write the probability distribution function for any biological species, whose total $G C$ content in percent in the exonic regions is $Y_{G C}$, as

$$
\begin{aligned}
f(n)= & \left(\alpha_{0}+\alpha_{1} Y_{G C}+\alpha_{2} Y_{G C}^{2}\right) e^{-\eta n}-n\left(\beta_{0}+\beta_{1} Y_{G C}\right. \\
& \left.+\beta_{2} Y_{G C}^{2}\right)+\gamma,
\end{aligned}
$$

where $\eta$ is obtained by solving Eq. (10). Of course we are not able to predict which codon occupies the $n$th rank. Finally, let us remark that the total exonic $G C$ content $Y_{G C}$ has to satisfy the consistency condition

$$
Y_{G C}=\frac{1}{3} \sum_{i \in I} d_{i} f(i)
$$

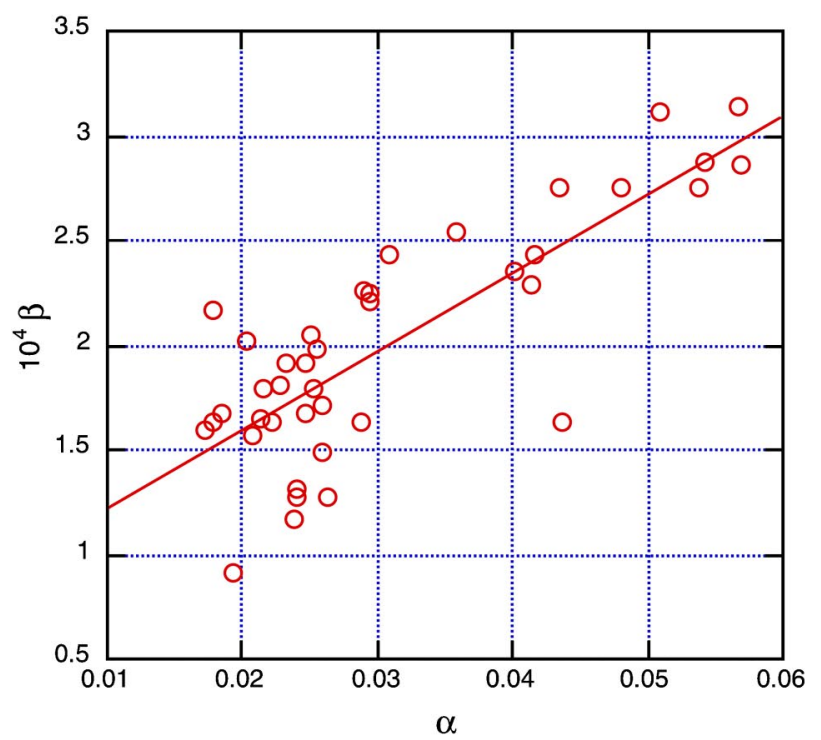

where the sum is over the set $I$ of integers to which the 56 codons containing $G$ and/or $C$ nucleotides belong and $d_{i}$ is the multiplicity of these nucleotides inside the $i$ th codon.

\section{AMINO-ACID RANK DISTRIBUTION}

It is natural to wonder if some kind of universality is also present in the rank distribution of amino acids. From the available data for codon usage, we can immediately compute (using the eukaryotic code) the frequency of appearance of any amino acid $F(n)(1 \leqslant n \leqslant 20)$ in the whole set of coding sequences. The calculated values as a function of the rank are satisfactorily fitted by a straight line

$$
F(n)=F_{0}-B n .
$$

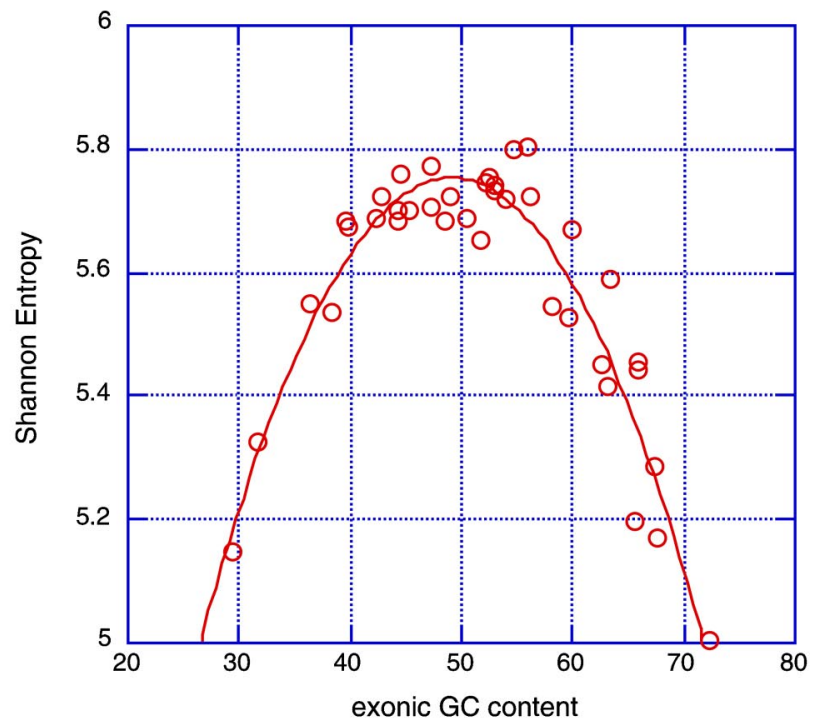

FIG. 10. Fits for the $\alpha$ and $\beta$ parameters and for the Shannon entropy. 
TABLE V. Values of the best-fit parameters for amino acids.

\begin{tabular}{|c|c|c|c|}
\hline Species & $10^{3} B$ & $F_{0}$ & $\chi^{2}$ \\
\hline Homo sapiens & 3.8 & 0.089 & 0.0072 \\
\hline Arabidopsis thaliana & 3.8 & 0.090 & 0.0068 \\
\hline Drosophila melanogaster & 3.5 & 0.087 & 0.0125 \\
\hline Caenorhabditis elegans & 3.3 & 0.084 & 0.0124 \\
\hline Mus musculus & 3.7 & 0.088 & 0.0087 \\
\hline Saccharomyces cerevisiae & 3.9 & 0.090 & 0.0121 \\
\hline Escherichia coli & 4.0 & 0.091 & 0.0115 \\
\hline Rattus norvegicus & 3.7 & 0.088 & 0.0084 \\
\hline Oryza sativa japonica & 4.1 & 0.093 & 0.0057 \\
\hline Schizosaccharomyces pombe & 3.8 & 0.089 & 0.0162 \\
\hline Bacillus subtilis & 4.0 & 0.091 & 0.0104 \\
\hline Pseudomonas aeruginosa & 4.9 & 0.101 & 0.0493 \\
\hline Mesorhizobium loti & 4.7 & 0.100 & 0.0215 \\
\hline Streptomyces coelicolor A3 & 5.6 & 0.109 & 0.0624 \\
\hline Sinorhizobium meliloti & 4.7 & 0.100 & 0.0188 \\
\hline Nostoc sp. PCC7120 & 4.0 & 0.092 & 0.0174 \\
\hline Oryza sativa & 3.9 & 0.091 & 0.0028 \\
\hline Agrobacterium tumefaciens str. C38 & 4.6 & 0.098 & 0.0144 \\
\hline Ralstonia solanacearum & 4.7 & 0.101 & 0.0351 \\
\hline Yersinia pestis & 4.0 & 0.092 & 0.0135 \\
\hline Methanosarcina acetivorans str. C2A & 4.1 & 0.092 & 0.0063 \\
\hline Vibrio cholerae & 3.9 & 0.091 & 0.0148 \\
\hline Escherichia coli $\mathrm{K} 12$ & 4.0 & 0.091 & 0.0154 \\
\hline Mycobacterium tuberculosis CDC1551 & 5.2 & 0.105 & 0.01121 \\
\hline Mycobacterium tuberculosis $\mathrm{H} 37 \mathrm{Rv}$ & 5.3 & 0.106 & - \\
\hline Bacillus halodurans & 4.0 & 0.091 & 0.0100 \\
\hline Clostridium acetobutylicum & 4.6 & 0.097 & 0.0076 \\
\hline Caulobacter crescentus CB15 & 5.1 & 0.104 & 0.0524 \\
\hline Gallus gallus & 3.6 & 0.088 & 0.0040 \\
\hline Synechocystis sp. PCC6803 & 4.1 & 0.093 & 0.0168 \\
\hline Sulfolobus solfataricus & 4.4 & 0.096 & 0.0143 \\
\hline Mycobacterium leprae & 4.9 & 0.101 & 0.0401 \\
\hline Brucella melitensis & 4.5 & 0.097 & 0.0142 \\
\hline Deinococcus radiodurans & 5.2 & 0.105 & 0.0679 \\
\hline Xenopus laevis & 3.5 & 0.086 & 0.0084 \\
\hline Listeria monocytogenes & 4.2 & 0.093 & 0.0088 \\
\hline Neurospora crassa & 4.0 & 0.091 & 0.0042 \\
\hline Clostridium perfringens & 4.6 & 0.098 & 0.0035 \\
\hline Leishmania major & 4.7 & 0.099 & 0.0367 \\
\hline Bos taurus & 3.6 & 0.087 & 0.0082 \\
\hline
\end{tabular}

The parameters $F_{0}$ and $B$ and the corresponding $\chi^{2}$ for the fits are reported in Table V. It is interesting to recall that the linear trend was noted, from the analysis of a small number of proteins, in 1955 by Gamow and Ycas [15]. A better fit can be obtained in general by using a third-degree polynomial; however, the range of the four parameters for this fit is larger than the range of the two-parameter fit. For a few biological species, we give below the parameters for the two fits (see also Fig. 11). The plots of the linear fits for a few biological species are given in Fig. 12. Note that the 21st point is just the contribution of the Stop codons, which of course has not been taken into account for the fits. One can remark that the most frequent amino acid is always above the line. This can be easily understood in the light of Eq. (4). Indeed, the most frequent amino acids get, in general, a contribution of the exponential term of Eq. (4) with a low value of $n$. 

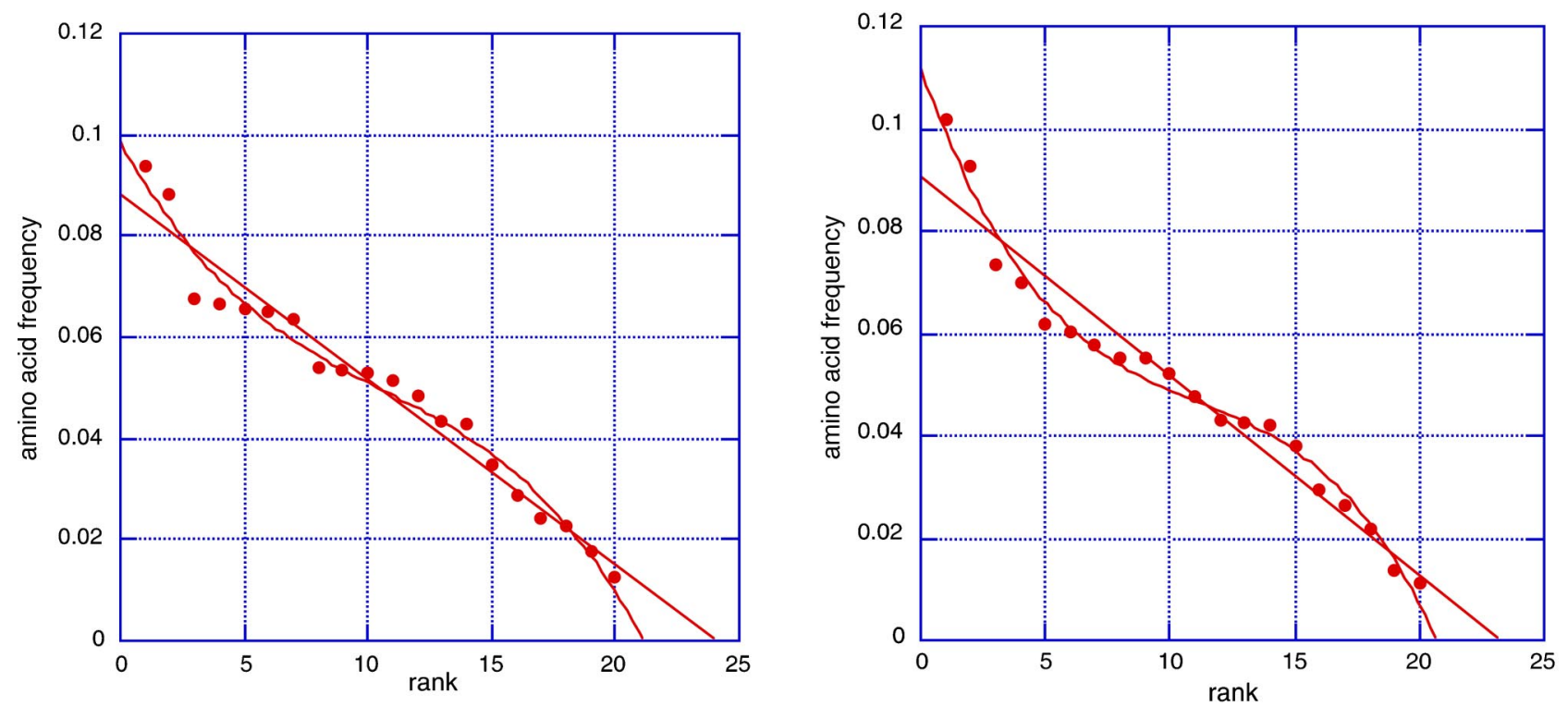

FIG. 11. Amino-acid frequency: linear vs cubic fits.
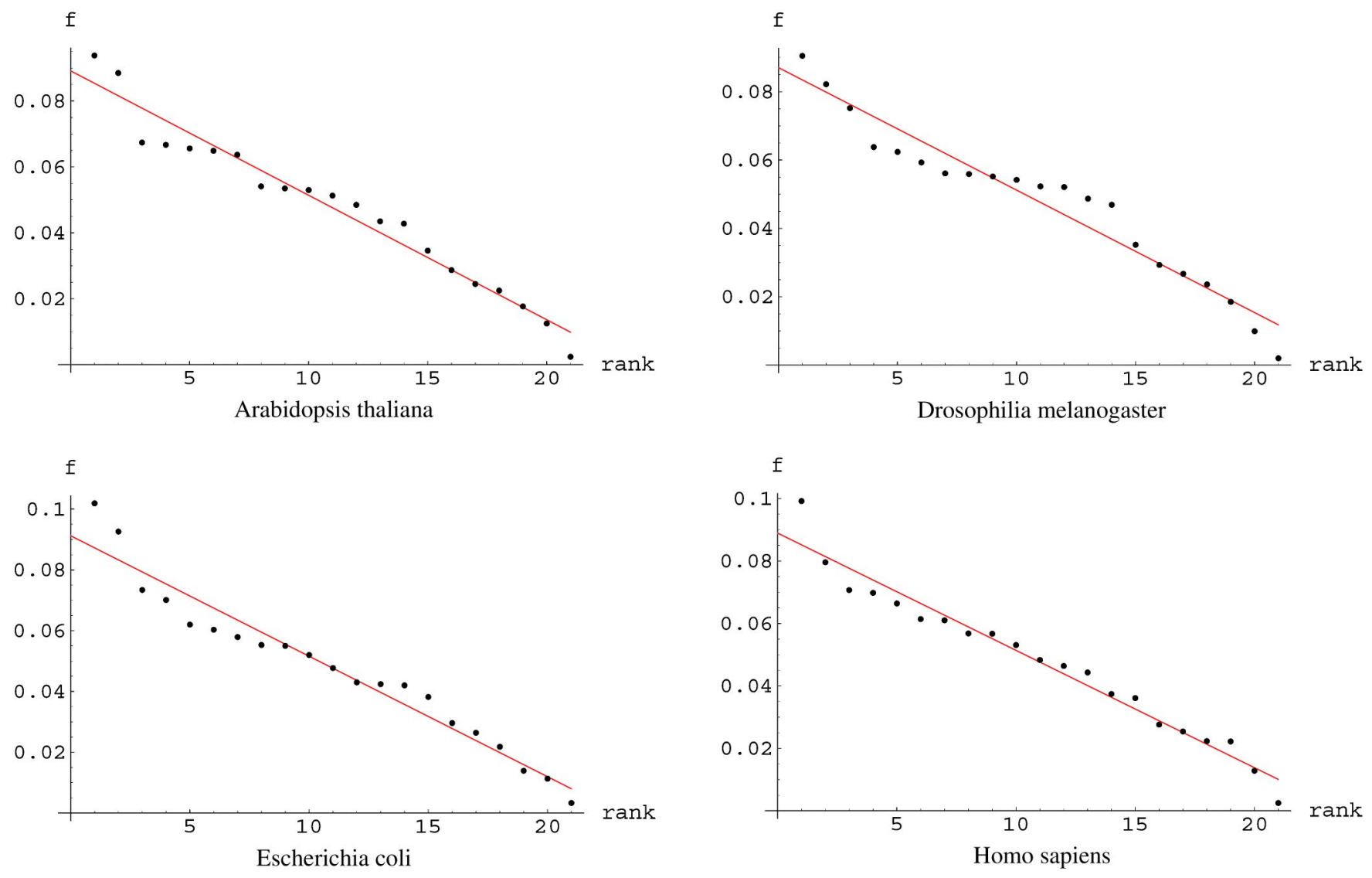

FIG. 12. Amino-acid rank distributions. 


\begin{tabular}{|c|c|c|}
\hline Species & Linear/cubic fits & $\chi^{2}$ \\
\hline \multirow[t]{2}{*}{ Homo sapiens } & lin. $f=0.087-0.0036 n$ & 0.0072 \\
\hline & $\begin{array}{l}\text { cub. } \\
\times 10^{-5} n^{3}\end{array}$ & 0.0055 \\
\hline \multirow[t]{2}{*}{ Arabidopsis thaliana } & lin. $f=0.088-0.0036 n$ & 0.0068 \\
\hline & $\begin{array}{l}\text { cub. } \\
\times 10^{-5} n^{3}\end{array} \quad f=0.099-0.0090 n+62 \times 10^{-5} n^{2}-1.95$ & 0.0049 \\
\hline \multirow[t]{2}{*}{ Drosophila melanogaster } & lin. $f=0.087-0.0036 n$ & 0.0125 \\
\hline & $\begin{array}{l}\text { cub. } \\
\times 10^{-5} n^{3}\end{array}$ & 0.0042 \\
\hline \multirow[t]{2}{*}{ Escherichia coli } & lin. $f=0.090-0.0039 n$ & 0.0115 \\
\hline & $\begin{array}{l}\text { cub. } \\
\times 10^{-5} n^{3}\end{array} \quad f=0.112-0.0136 n+105 \times 10^{-5} n^{2}-3.1$ & 0.0067 \\
\hline
\end{tabular}

Of course, the frequency of an amino acid is given by the sum of the frequencies of its encoding codons given by Eq. (4). If the ranks of the encoding codons were completely random, we would not expect their sum to take equally spaced values, as is the case in a regression line. Therefore, we can infer, for the biological species whose amino-acid frequency is very well fitted by a line, the existence of some functional constraints on the codon usage.

We report in Table VI the distribution of the amino acids for the different biological species. There is no clear correlation between the rank of the codons and the rank of the encoded amino acids. As has been previously remarked, in many species three of the four most used codons encode for doublets which are generally less used than the five quartets and the three sextets. ${ }^{3}$ The statement is illustrated by Fig. 13, where we plot, for Homo sapiens, the frequencies of the codons according to the rank of the encoded amino acids, indicating for each amino acid the rank of the corresponding codons. In the legend, for each amino acid, "codon 1" means the most used codon, "codon 2" the next most used codon, and so on.

However, the behavior predicted by Eq. (4) fits the experimental data very well, while the shape of the distribution of amino acids seems more sensible for biological species. In fact, one can remark in many plots of the amino-acid distributions (see, e.g., Fig. 12) the existence of one or two plateaus, which obviously indicate equal probabilities of use for some amino acids. Presently, we do not have any argument to explain the uniform distribution of amino acids from the ranked distribution of the corresponding codons.

\section{CONSEQUENCES OF PROBABILITY DISTRIBUTION}

We now derive a few consequences of Eq. (4). In the following, we denote by $y$ the local exonic $G C$ content (i.e., for coding sequences of genes) for a given biological species. Let us assume that the exonic $G C$ content of a biological species is essentially comprised in the interval $y_{1}-y_{0}=\Delta$

\footnotetext{
${ }^{3}$ Here and elsewhere, the words doublet, quartet, sextet, etc., refer to the group of (synonymous) codons coding for the same amino acid.
}

(e.g., for Homo sapiens $y_{0}=35 \%$ and $y_{1}=70 \%$ ). We can write

$$
f(n)=\frac{1}{\Delta} \int_{y_{0}}^{y_{1}} f(y, n) d y .
$$

Since the left-hand side of the above equation has the form given by Eq. (4) for any $n$ and for any biological species, if we do not want to invoke some "fine-tuning" in the integrand function $f(y, n)$, we have to assume that

$$
f(y, n)=a(y) e^{-\eta n}-b(y) n+\gamma
$$

with the condition

$$
\alpha=\frac{1}{\Delta} \int_{y_{0}}^{y_{1}} a(y) d y, \quad \beta=\frac{1}{\Delta} \int_{y_{0}}^{y_{1}} b(y) d y .
$$

As a consequence, we predict that the codon usage probability is the same for any codon in any exonic genic region with the same $G C$ content. The form of the $a(y)$ and $b(y)$ functions is yet undetermined. For Homo sapiens, we remark that the total exonic $G C$ content $Y_{G C}$ is, in a very good approximation, equal to the mean value of the interval $\left[y_{0}, y_{1}\right]$. Therefore, inserting Eqs. (14) and (15) into Eq. (12), we derive the result that the functions $a(y)$ and $b(y)$ have to be linear functions of $y$. This theoretical derivation is in accordance with the conclusions of Zeeberg [16] obtained by an analysis of 7357 genes. On a quantitative level, using the numerical linear fits of Zeeberg, we find a very good agreement with our calculations. Note that this result is not in contradiction with Eq. (11), since the previous analysis is valid for the fixed value of the exonic GC content for Homo sapiens. For bacteria, the range of variation $\Delta$ of the local exonic $G C$ content is very small. Therefore we expect the functions $a(y)$ and $b(y)$ to have the same shape as the functions $\alpha$ and $\beta$ given in Eqs. (7) and (8). Hence the functions $\alpha$ and $\beta$ depend on the biological species.

We compute the Shannon entropy, given by

$$
S=-\sum_{n} f(n) \log _{2} f(n)
$$


TABLE VI. Type of amino acids of the observed rank distribution.

\begin{tabular}{|c|c|c|c|c|c|c|c|c|c|c|c|c|c|c|c|c|c|c|c|c|}
\hline \multirow[b]{2}{*}{ Species } & \multicolumn{20}{|c|}{ Rank } \\
\hline & 1 & 2 & 3 & 4 & 5 & 6 & 7 & 8 & 9 & 10 & 11 & 12 & 13 & 14 & 15 & 16 & 17 & 18 & 19 & 20 \\
\hline Homo sapiens & Leu & Ser & Ala & Glu & Gly & Val & Pro & Lys & Arg & Thr & Asp & Gln & Ile & Phe & Asn & Tyr & His & Met & Cys & Trp \\
\hline Mus musculus & Leu & Ser & Ala & Gly & Glu & Val & Pro & Lys & Arg & Thr & Asp & Ile & Gln & Phe & Asn & Tyr & His & Cys & Met & $\operatorname{Trp}$ \\
\hline Rattus norvegicus & Lue & Ser & Ala & Glu & Gly & Val & Pro & Lys & Thr & Arg & Asp & Ile & Gln & Phe & Asn & Tyr & His & Met & Cys & $\operatorname{Trp}$ \\
\hline Gallus gallus & Leu & Ser & Glu & Ala & Gly & Lys & Val & Pro & Thr & Arg & Asp & Ile & Gln & Asn & Phe & Tyr & His & Met & Cys & $\operatorname{Trp}$ \\
\hline Xenopus laevis & Leu & Ser & Glu & Lys & Ala & Gly & Val & Pro & Thr & Asp & Arg & Ile & Gln & Asn & Phe & Tyr & Met & His & Cys & Trp \\
\hline Bos taurus & Leu & Ser & Ala & Gly & Glu & Val & Lys & Pro & Thr & Arg & Asp & Ile & Gln & Phe & Asn & Tyr & Cys & His & Met & $\operatorname{Trp}$ \\
\hline Arabidopsis thaliana & Leu & Ser & Val & Glu & Gly & Ala & Lys & Asp & Arg & Ile & Thr & Pro & Asn & Phe & Gln & Tyr & Met & His & Cys & $\operatorname{Trp}$ \\
\hline Oryza sativa japonica & Ala & Leu & Gly & Ser & Arg & Val & Glu & Pro & Asp & Thr & Lys & Ile & Phe & Gln & Asn & His & Tyr & Met & Cys & $\operatorname{Trp}$ \\
\hline Oryza sativa & Ala & Leu & Gly & Ser & Arg & Val & Glu & Pro & Asp & Lys & Thr & Ile & Phe & Gln & Asn & Tyr & His & Met & Cys & Trp \\
\hline Neurospora crassa & Ala & Leu & Ser & Gly & Glu & Pro & Arg & Thr & Val & Asp & Lys & Ile & Gln & Asn & Phe & Tyr & His & Met & $\operatorname{Trp}$ & Cys \\
\hline Drosophila melanogaster & Leu & Ser & Ala & Glu & Gly & Val & Lys & Thr & Arg & Pro & Asp & Gln & Ile & Asn & Phe & Tyr & His & Met & Cys & Trp \\
\hline Caenorhabditis elegans & Leu & Ser & Glu & Lys & Ala & Val & Ile & Thr & Gly & Arg & Asp & Asn & Phe & Pro & Gln & Tyr & Met & His & Cys & $\operatorname{Trp}$ \\
\hline ıania major & Ala & Leu & Ser & Arg & Val & Gly & Thr & Pro & Glu & Asp & Gln & Lys & Ile & His & Phe & Asn & Tyr & Met & Cys & $\operatorname{Trp}$ \\
\hline cerevis & Leu & Ser & Lys & Ile & Glu & Asn & Thr & Asp & Val & Ala & Gly & Arg & Phe & Pro & Gln & Tyr & His & Met & Cys & $\operatorname{Trp}$ \\
\hline Schizosacch. pombe & Leu & Ser & Glu & Lys & Ala & Ile & Val & Thr & Asp & Asn & Gly & Arg & Pro & Phe & Gln & Tyr & His & Met & Cys & $\operatorname{Trp}$ \\
\hline Escherichia coli & Leu & Ala & Gly & Val & Ser & Ile & Glu & Thr & Arg & Asp & Lys & Gln & Pro & Asn & Phe & Tyr & Met & His & $\operatorname{Trp}$ & Cys \\
\hline ubtilis & Leu & Ala & Ile & Glu & Lys & Gly & Val & Ser & Thr & Asp & Phe & Arg & Asn & Gln & Pro & Tyr & Met & His & Trp & Cys \\
\hline Pseudom. aeruginosa & Leu & Ala & Gly & Arg & Val & Glu & Ser & Asp & Pro & Gln & Thr & Ile & Phe & Lys & Asn & Tyr & His & Met & $\operatorname{Trp}$ & Cys \\
\hline Mesorhizobium loti & Ala & Leu & Gly & Val & Arg & Ser & Asp & Ile & Glu & Thr & Pro & Phe & Lys & Gln & Asn & Met & Tyr & His & $\operatorname{Trp}$ & Cys \\
\hline licolor A3 & Ala & Leu & Gly & Val & Arg & Pro & Thr & Asp & Glu & Ser & Ile & Phe & Gln & His & Lys & Tyr & Asn & Met & $\operatorname{Trp}$ & Cys \\
\hline Sinorhizobium meliloti & la & Leu & Gly & Val & Arg & & er & Ile & Asp & Thr & ro & Phe & Lys & Gln & Asn & Met & Tyr & His & Trp & Cys \\
\hline Nostoo sp. PCC7120 & Leu & Ala & Ile & Val & Gly & Ser & Glu & Thr & Gln & Arg & Lys & Asp & Pro & Asn & Phe & Tyr & His & Met & $\operatorname{Trp}$ & Cys \\
\hline Agrobact. tumefaciens & Ala & Leu & Gly & Val & Arg & Ser & Glu & Ile & Asp & Thr & Pro & Phe & Lys & Gln & Asn & Met & Tyr & His & $\operatorname{Trp}$ & Cys \\
\hline Ralstonia solanacearum & Ala & Leu & Gly & Val & Arg & Thr & $\mathrm{sp}$ & Pro & Ser & Glu & Ile & $\ln$ & Phe & Lys & Asn & Tyr & His & Met & $\operatorname{Trp}$ & Cys \\
\hline Yersinia pestis & Leu & Ala & Gly & Val & Ser & Ile & Glu & Thr & Arg & Asp & Gln & Lys & Pro & Asn & Phe & Tyr & Met & His & Trp & Cys \\
\hline Methanosarc. acetivorans & Leu & Glu & Ile & Gly & Ser & Ala & Val & Lys & Thr & Asp & Arg & Asn & Phe & Pro & Tyr & Gln & Met & His & Cys & $\operatorname{Trp}$ \\
\hline Vibrio cholerae & Leu & Ala & Val & Gly & Ser & Ile & Glu & Thr & Asp & Gln & Lys & Arg & Phe & Asn & Pro & Tyr & Met & His & $\operatorname{Trp}$ & Cys \\
\hline Escherichia coli $\mathrm{K} 12$ & Leu & Ala & Gly & Val & Ile & Ser & Glu & Arg & Thr & Asp & Gln & Pro & Lys & Asn & Phe & Tyr & Met & His & Trp & Cys \\
\hline Mycobact. tuber. CDC1551 & Ala & Leu & Gly & Val & Arg & Thr & Pro & Asp & Ser & Glu & Ile & Gln & Phe & Asn & His & Tyr & Lys & Met & $\operatorname{Trp}$ & Cys \\
\hline Mycobact. tuber. H37Rv & Ala & Gly & Leu & Val & Arg & Thr & Asp & Pro & Ser & Glu & Ile & Gln & Phe & Asn & His & Tyr & Lys & Met & $\operatorname{Trp}$ & Cys \\
\hline Bacillus halodurans & Leu & Glu & Val & Ala & Gly & Ile & Lys & Ser & Thr & Asp & Arg & Phe & Gln & Pro & Asn & Tyr & Met & His & $\operatorname{Trp}$ & Cys \\
\hline Clostridium acetobutylicum & Ile & Lys & Leu & Ser & Glu & Val & Asn & Gly & Ala & Asp & Thr & Phe & Tyr & Arg & Pro & Met & Gln & His & Cys & Trp \\
\hline Caulobacter crescentus CB15 & Ala & Leu & Gly & Val & Arg & Asp & Pro & Glu & Thr & Ser & Ile & Phe & Lys & Gln & Asn & Met & Tyr & His & Trp & Cys \\
\hline Synechocystis sp. PCC6803 & Leu & Ala & Gly & Val & Ile & Glu & Ser & Gln & Thr & Pro & Arg & Asp & Lys & Asn & Phe & Tyr & Met & His & $\operatorname{Trp}$ & Cys \\
\hline Sulfolobus solfatarcus & Leu & Ile & Lys & Val & Glu & Ser & Gly & Ala & Asn & Tyr & Arg & Thr & Asp & Phe & Pro & Gln & Met & His & $\operatorname{Trp}$ & Cys \\
\hline Mycobacterium leprae & Ala & Leu & Val & Gly & Arg & Thr & Ser & Asp & Pro & Glu & Ile & Gln & Phe & Lys & Asn & His & Tyr & Met & $\operatorname{Trp}$ & Cys \\
\hline Brucella melitensis & Ala & Leu & Gly & Val & Arg & Ile & Glu & Ser & Asp & Thr & Pro & Lys & Phe & Gln & Asn & Met & Tyr & His & $\operatorname{Trp}$ & Cys \\
\hline Deinococcus radiodurans & Ala & Leu & Gly & Val & Arg & Pro & Thr & Glu & Ser & Asp & Gln & Ile & Phe & Lys & Asn & Tyr & His & Met & Trp & Cys \\
\hline Listeria monocytogenes & Leu & Ile & Ala & Glu & Lys & Val & Gly & Thr & Ser & Asp & Asn & Phe & Arg & Pro & Gln & Tyr & Met & His & $\operatorname{Trp}$ & Cys \\
\hline Clostridium perfringens & Ile & Leu & Lys & Glu & Gly & Val & Asn & Ser & Asp & Ala & Thr & Phe & Tyr & Arg & Pro & Met & Gln & His & Cys & $\operatorname{Trp}$ \\
\hline
\end{tabular}

for the codons of a biological species and plot it versus the total exonic GC content; see Fig. 10. The Shannon entropy is rather well fitted by a parabola:

$$
S=2.2186+0.144 Y_{G C}-0.00146 Y_{G C}^{2}, \quad \chi^{2}=0.0315
$$

Note that the parabola has its apex for $y \approx 0.50$, which is expected for the behavior of the Shannon entropy for two variables (here $G C$ and its complementary $A U$ ).
The same behavior has been shown by analogous computations made by Zeeberg [16] for Homo sapiens. So it seems that the entropy in the gene coding sequences and in the total exonic region as functions of the exonic $G C$ content show the same pattern.

In conclusion, the distribution of the experimental codon probabilities for a large total exonic region of several biological species has been very well fitted by the law of Eq. (4). The spectrum of the distribution is universal, but the codon, which occupies a fixed level, depends on the biological species. Indeed, a more detailed analysis shows that, for 


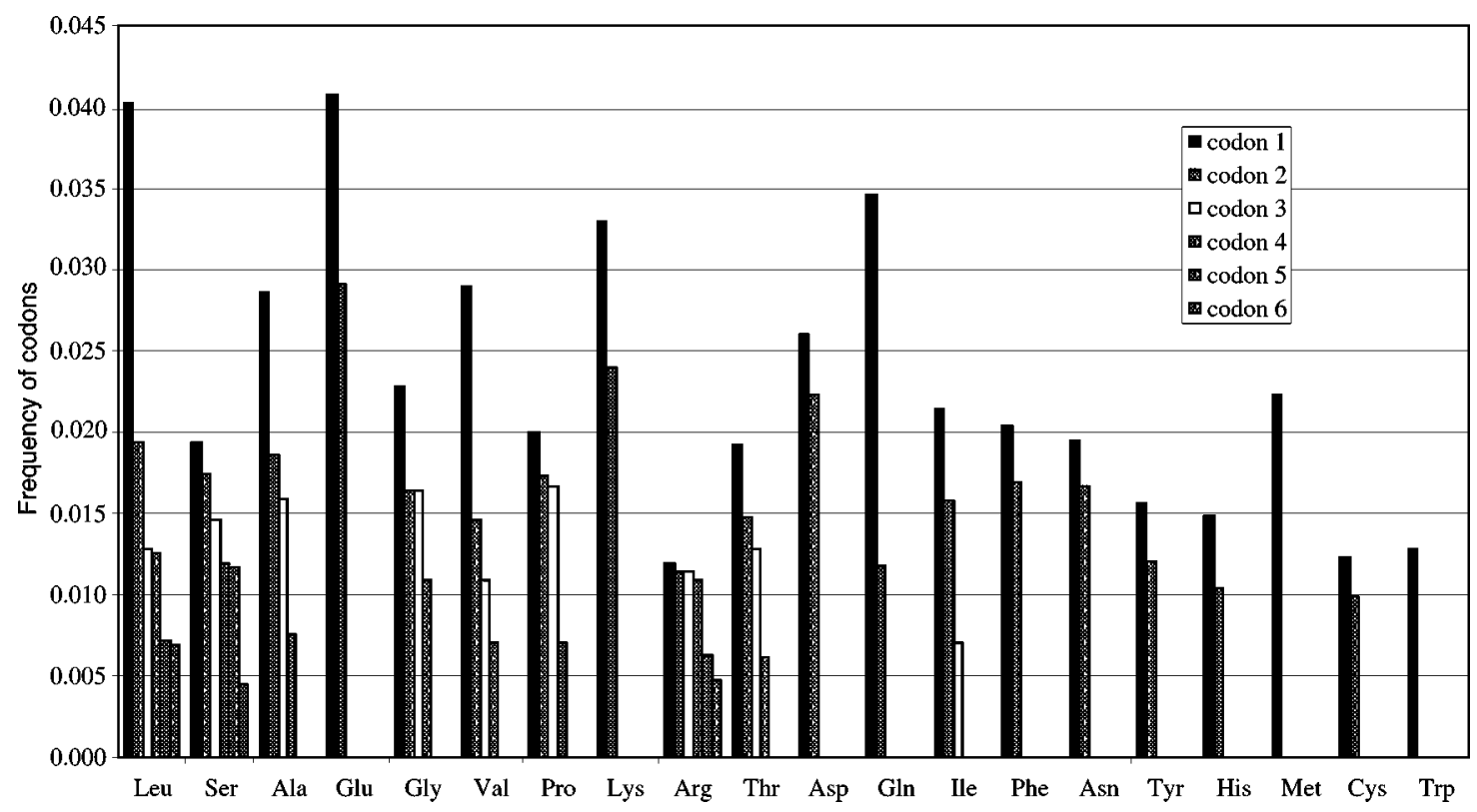

FIG. 13. Amino-acid and codon ranked distributions for Homo sapiens.

close biological species, e.g., vertebrates, a fixed codon occupies almost the same position in $f(n)$, while for distant biological species the codons occupy very different positions in the rank distribution. We have also derived that the codon frequency for any gene region is the same for fixed biological species and fixed $G C$ content. Entropy analysis has shown that the behavior observed in genes with different $G C$ content for the same biological species is very similar to that shown by the total exonic region with different $G C$ content for different biological species.

\section{ACKNOWLEDGMENTS}

The authors would like to thank A. Arneodo for fruitful discussions. A.S. is indebted to the Université de Savoie for financial support and LAPTH for its kind hospitality.
[1] G. K. Zipf, Human Behaviour and the Principle of Least Effort (Addison-Wesley, Cambridge, MA, 1949).

[2] R. N. Mantegna, S. V. Buldyrev, A. L. Goldberger, S. Havlin, C.-K. Peng, M. Simons, and H. E. Stanley, Phys. Rev. Lett. 73, 3169 (1994).

[3] A. Czirok, R. N. Mantegna, S. Havlin, and H. E. Stanley, Phys. Rev. E 52, 446 (1995).

[4] R. Israeloff, M. Kagalenko, and K. Chan, Phys. Rev. Lett. 76, 1976 (1996); S. Bonhoeffer, A. V. M. Herz, M. C. Boerlijst, S. Nee, M. A. Nowak, and R. M. May, ibid. 76, 1977 (1996); R. F. Voss, ibid. 76, 1978 (1996); R. N. Mantegna, S. V. Buldyrev, A. L. Goldberger, S. Havlin, C.-K. Peng, M. Simons, and H. E. Stanley, ibid. 76, 1979 (1996).

[5] C. Martindale and A. K. Konopka, Comput. Chem. (Oxford) 20, 35 (1996).

[6] G. U. Yule, Philos. Trans. R. Soc. London, Ser. B 213, 21 (1924); The Statistical Study of Literary Vocabulary (Cam- bridge University Press, Cambridge, U.K., 1944).

[7] W. Li and Y. Yang, J. Theor. Biol. 219, 539 (2002).

[8] C. Furusawa and K. Kaneko, Phys. Rev. Lett. 90, 088102 (2003).

[9] V. A. Kuznetsov, Signal Process. 83, 889 (2003).

[10] A. Som, S. Chattapadhyay, J. Chakrabarti, and D. Bandyopadhyay, Phys. Rev. E 63, 051908 (2001).

[11] C. E. Shannon, Bell Syst. Tech. J. 27, 623 (1948).

[12] Y. Nakamura, T. Gojobori, and T. Ikemura, Nucleic Acids Res. 28, 292 (2000).

[13] L. Frappat, A. Sciarrino, and P. Sorba, J. Biol. Phys. 17, 1 (2001).

[14] R. D. Knight, S. J. Freeland, and L. F. Landweber, Genome Biol. 2, 1 (2001).

[15] G. Gamow and M. Ycas, Proc. Natl. Acad. Sci. U.S.A. 41, 1011 (1955).

[16] B. Zeeberg, Genome Res. 12, 944 (2002). 